\title{
A rare case of supraspinatus tendon rupture
}

\author{
Leonie Strobbe • Roger J. M. Brüggemann • \\ Peter J. Donnelly $•$ Nicole M. A. Blijlevens
}

Received: 14 March 2011 / Accepted: 15 March 2011 /Published online: 29 March 2011

(C) The Author(s) 2011. This article is published with open access at Springerlink.com

\begin{abstract}
Dear Editor,
A 66-year-old man was treated for acute myelomonocytic leukemia with mitoxantrone, cytarabine, and etoposide. As it is our practice, we gave him $500 \mathrm{mg}$ ciprofloxacin every $12 \mathrm{~h}$ per os for antibacterial prophylaxis. Two weeks later, he complained of pain in both shoulders which worsened until he could no longer raise his arms. He also had pain in his hips and knees, but the Achilles tendons were not involved. There were no signs of inflammation, but palpation of the insertion point of the supraspinatus tendon proved painful. Ultrasound examination showed a bilateral partial rupture of the supraspinatus tendon and subscapularis tendinopathy (Fig. 1). We suspected ciprofloxacin toxicity so we stopped the drug and he was given analgesics and physiotherapy. The pain resolved completely within a few days; however, full functional recovery took several months. We did not re-challenge with ciprofloxacin as the side effects had been so severe.
\end{abstract}

L. Strobbe $(\bowtie) \cdot$ R. J. M. Brüggemann • P. J. Donnelly $•$

N. M. A. Blijlevens

Department of Hematology,

Radboud University Nijmegen Medical Centre,

6500 HB Nijmegen, The Netherlands

e-mail: L.strobbe@hemat.umcn.nl
Antibiotic prophylaxis reduces infections and enhances survival when given to high-risk patients receiving chemotherapy for acute leukemia or stem cell transplant. Current guidelines recommend prophylaxis be given from the start of chemotherapy until bone marrow recovery when the expected duration of neutropenia is more than 7 days $[1,2]$. Fluoroquinolones, which provide a broad antimicrobial spectrum, are generally well tolerated and have a good safety profile. However, they can cause tendinitis and other forms of tendon damage in $10-15$ per 100,000 prescriptions, a rate that increases with older age, renal dysfunction, and corticosteroid use. The onset of symptoms is typically within 2 weeks after starting treatment, but symptoms can occur within $2 \mathrm{~h}$ after the first dose until 6 months after cessation of treatment. The Achilles tendon is affected in $90 \%$ cases, almost half involving both ankles and the tendon ruptures in approximately $50 \%$ of the cases. Other sites of tendon injury have been reported but are rare. Recovery usually takes 1-2 months requiring rest and immobilization and surgery is seldom needed [3, 4]. How fluoroquinolones induce tendon injury is not understood.

Our patient's age appears to have been the only risk factor as he had not used corticosteroids and did not have renal dysfunction. In July 2008, the FDA added a black box warning to alert doctors and patients of the raised risk of tendinitis and tendon rupture associated with fluoroquinolones [5]. As clinicians will be treating an increasing 


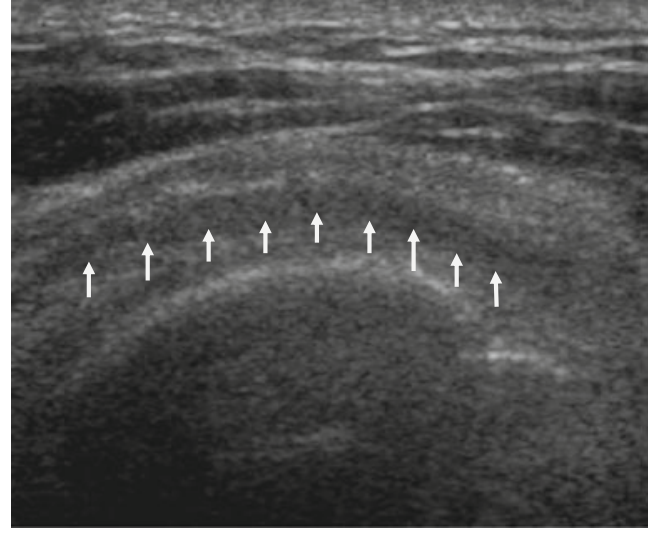

Fig. 1 Ultrasound of the left supraspinatus tendon. The ultrasound shows a partial longitudinal rupture of the supraspinatus tendon (white arrows)

number of elderly patients for hematological malignancies, it is very important for them to recognize these symptoms and switch to an alternative treatment before tendon rupture occurs.
Open Access This article is distributed under the terms of the Creative Commons Attribution Noncommercial License which permits any noncommercial use, distribution, and reproduction in any medium, provided the original author(s) and source are credited.

\section{References}

1. Segal BH, Freifeld AG (2007) Antibacterial prophylaxis in patients with neutropenia. J Natl Compr Canc Netw 5:235-242

2. Bucaneve G, Castagnola E, Viscoli C, Leibovici L, Menichetti F (2007) Guidelines from the First European Conference on Infections in Leukaemia: ECIL1 quinolone prophylaxis for bacterial infections in afebrile high risk neutropenic patients. EJC Supplements 5:5-12

3. Karistinos A, Paulos LE (2007) "Ciprofloxacin-induced" bilateral rectus femoris tendon rupture. Clin J Sport Med 17:406-407

4. Khaliq Y, Zhanel GG (2003) Fluorochinolon-associated tendinopathy: a critical review of the literature. Clin Infect Dis 36:14041410

5. Tanne JH (2008) FDA adds "black box" warning to fluoroquinolone antibiotics. BMJ 337:a816 